\title{
A New Inverse Rayleigh Distribution: Properties and Application
}

\author{
A.S. Malik $^{1^{*}}$, S.P. Ahmad ${ }^{2}$ \\ ${ }^{1,2}$ Department of Statistics, University of Kashmir, Srinagar, India \\ "Corresponding Author: aaliyasayeed2@gmail.com, Tel.:+91-8494059449
}

Available online at: www.isroset.org

Received:06/Sept/2018, Accepted:02/Oct/2018, Online: 31/Oct/2018

\begin{abstract}
In this paper, a new generalization of inverse Rayleigh Distribution known as Alpha Power inverse Rayleigh distribution is proposed using Alpha Power Transformation. Some mathematical properties of the Alpha Power inverse Rayleigh distribution have been derived and the parameters are estimated using Maximum Likelihood estimation method. Two real life data sets have been incorporated to illustrate the applicability of the proposed distribution.
\end{abstract}

Keywords - Alpha power inverse Rayleigh Distribution, Alpha Power Transformation, Entropy, Reliability, MLE.

\section{INTRODUCTION}

Inverse Rayleigh Distribution (IRD) was recommended by Trayer [1].IRD finds massive applicability in survival analysis. Voda [2] also studied various properties of IRD.

The pdf of IRD is given by

$$
g(x)=\left(\frac{2 \lambda}{x^{3}}\right) \exp \left(-\frac{\lambda}{x^{2}}\right) \quad x, \lambda>0 .
$$

The corresponding cdf is given by

$$
G(x)=\exp \left(-\frac{\lambda}{x^{2}}\right) \quad x, \lambda>0 \text {. }
$$

In this paper our motive is to generalize IRD to increase its flexibility so that more and more data can be analysed using this distribution. Among the diverse generalization techniques available in literature, the generalization of our interest is Alpha Power Transformation (APT) suggested by Mahdavi and Kundu [3] which enables to enhance the flexibility of probability distribution by incorporating an extra parameter. This transformation has produced a number of flexible distribution. Few among them are: Alpha power Weibull distribution: Properties and applications by Alzaatreh et al. [4], Alpha Power Rayleigh Distribution and its Application to Life Time Data by Malik and Ahmad [5] etc. The APT of a random variable x with cdf $G(x)$ and pdf $g(x)$ is defined as

$G_{A P T}(x)=\left\{\begin{array}{ll}\frac{\alpha^{G(x)}-1}{\alpha-1} & ; \alpha>0, \alpha \neq 1 \\ G(x) & ; \alpha=1\end{array}\right.$.
The corresponding pdf is given as

$$
g_{A P T}(x)= \begin{cases}\frac{\log \alpha}{\alpha-1} g(x) \alpha^{G(x)} & ; \alpha>0, \alpha \neq 1 \\ g(x) & ; \alpha=1\end{cases}
$$

In this context we propose Alpha Power Inverse Rayleigh Distribution (APIRD), a flexible extension of IRD based on Alpha Power Transformation (APT) proposed by Mahdavi and Kundu [3]. The framework of paper is unfolded as: section II has been committed for deriving pdf and cdf of the proposed distribution. In section III-VI, the reliability measures and various properties of APIRD are obtained. The expressions for information measures, stress strength reliability and order statistics is obtained in section VII, VIII and IX respectively. In section XI, the parameter estimation has been carried out. In section XII the application of the APIRD is debated using real life examples and finally some conclusions and discussions are given in section XII and XIV.

\section{APIRD}

The cdf of APIRD obtained by substituting eqn. (2) in eqn. (3) is given by

$$
G_{A P T}(x)=\left\{\begin{array}{ll}
\frac{\left(\alpha^{e^{-\frac{\lambda}{x^{2}}}}-1\right)}{(\alpha-1)} & ; \alpha \neq 1 \\
e^{-\frac{\lambda}{x^{2}}} & ; \alpha=1
\end{array} \quad x>0, \lambda>0, x>0 .\right.
$$


The corresponding pdf of APIRD is given by

$$
g_{A P T}(x)=\left\{\begin{array}{ll}
\frac{\log \alpha}{(\alpha-1)} \frac{2 \lambda}{x^{3}} e^{-\frac{\lambda}{x^{2}}} \alpha^{e^{-\frac{\lambda}{x^{2}}}} & ; \alpha \neq 1 \\
\frac{2 \lambda}{x^{2}} e^{-\frac{\lambda}{x^{2}}} & ; \alpha=1
\end{array} .\right.
$$

The graph of pdf and cdf function for $\lambda=1$ and distinct values $\alpha$ is given in Figure 1 and 2 respectively.

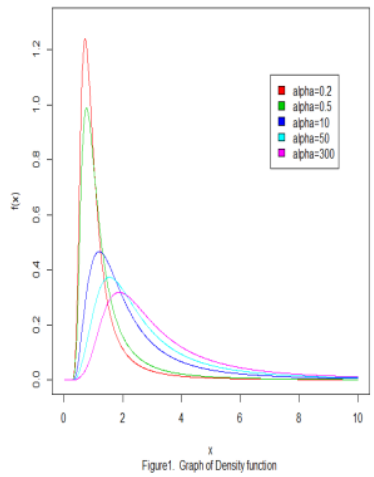

III. RELIABILITY ANALYSIS

\section{Survival function}

The survival function of APIRD is given as

$$
s_{A P T}(x)= \begin{cases}\frac{\alpha\left(1-\alpha^{e^{-\frac{\lambda}{x^{2}}}}\right)}{(\alpha-1)} & ; \alpha \neq 1 \\ 1-e^{-\frac{\lambda}{x^{2}}} & ; \alpha=1\end{cases}
$$

\section{Hazard Rate (hrf)}

The hrf of APIRD is given as

$$
h_{A P T}(x)= \begin{cases}\frac{\log \alpha}{\left(1-\alpha^{e^{-\frac{\lambda}{x^{2}}}-1}\right)} \frac{2 \lambda}{x^{3}} e^{-\frac{\lambda}{x^{2}}} \alpha^{e^{-\frac{\lambda}{x^{2}}}-1} & ; \alpha \neq 1 \\ \frac{2 \lambda}{x^{2}\left(1-e^{-\frac{\lambda}{x^{2}}}\right)} e^{-\frac{\lambda}{x^{2}}} & ; \alpha=1\end{cases}
$$

For rest of the paper we study $\alpha \neq 1$ as for $\alpha=1$ APIRD reduces to IRD.

The graph of reliability function and hrf of APIRD for $\lambda=1$ and distinct values of $\alpha$ is given in Figure 3 and 4 respectively.
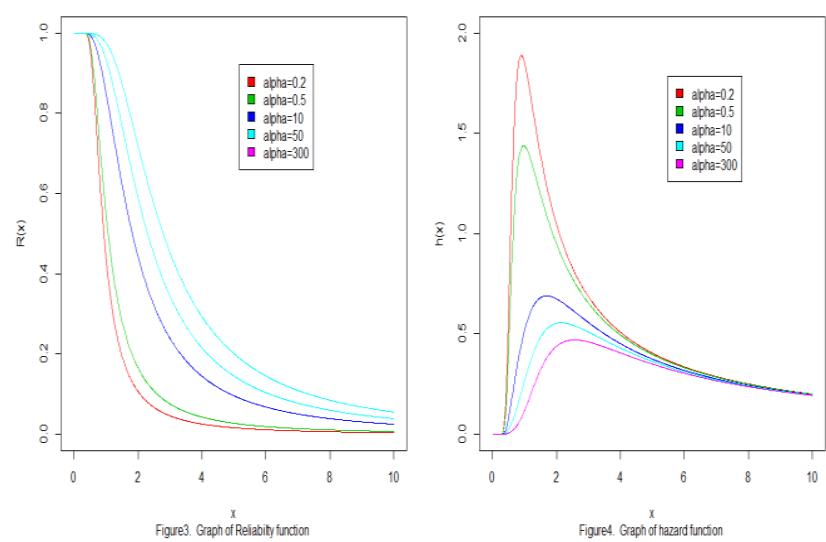

\section{MOMENTS}

The moments of APIRD can be obtained as

$\mu_{r}^{\prime}=\int_{0}^{\infty} x^{r} \frac{\log \alpha}{(\alpha-1)} \frac{2 \lambda}{x^{3}} e^{-\frac{\lambda}{x^{2}}} \alpha^{e^{-\frac{\lambda}{x^{2}}}} d x$

Using the expansion

$$
a^{u}=\sum_{k=0}^{\infty} \frac{(\log a)^{k} u^{k}}{k !} .
$$

We get

$\mu_{r}^{\prime}=\sum_{k=0}^{\infty} \frac{\lambda^{\frac{r}{2}}(\log \alpha)^{k+1}}{(\alpha-1) k !} \frac{\Gamma\left(1-\frac{r}{2}\right)}{(k+1)^{1-\frac{r}{2}}}$

The above equation holds for $r<2$. Putting $r=1$ in eqn. (9) we obtain the mean of APIRD

$$
\text { Mean }=\sum_{k=0}^{\infty} \frac{\lambda^{\frac{1}{2}}(\log \alpha)^{k+1}}{(\alpha-1) k !} \frac{\Gamma\left(\frac{1}{2}\right)}{(k+1)^{\frac{1}{2}}} \text {. }
$$

\section{MOMENT GENERATING FUNCTION (MGF.)}

The mgf. Of APIRD can be expressed as

$$
M_{X}(t)=\sum_{r=0}^{\infty} \sum_{k=0}^{\infty} \frac{t^{r}}{r !} \frac{\lambda^{\frac{r}{2}}(\log \alpha)^{k+1}}{(\alpha-1) k !} \frac{\Gamma\left(1-\frac{r}{2}\right)}{(k+1)^{1-\frac{r}{2}}} \text {. }
$$

\section{MEAN RESIDUAL LIFE (MRL) AND MEAN} WAITING TIME (MWT)

The MRL is defined as 


$$
\mu_{A P T}(t)=\frac{1}{s_{A P T}(t)}\left[E(t)-\int_{0}^{t} x g_{A P T}(x) d x\right]-t .
$$

Using eqn. (4), (7) and (10) in eqn. (11) we get

$$
\begin{aligned}
& \mu_{A P T}(t)=\frac{1}{\frac{\alpha\left(1-\alpha^{e^{-\frac{\lambda}{x^{2}}}-1}\right)}{(\alpha-1)}}\left[\sum_{k=0}^{\infty}\left(\frac{\lambda}{(k+1)}\right)^{\frac{1}{2}} \frac{(\log \alpha)^{k+1}}{(\alpha-1) k !} \Gamma\left(\frac{1}{2}\right)-\right. \\
& \mu_{A P T}(t)=\frac{\left.\sum_{k=0}^{\frac{1}{2}}\left(\frac{\lambda}{(k+1)}\right)^{\frac{1}{2}} \frac{(\log \alpha)^{k+1}}{(\alpha-1) k !} \Gamma\left(\frac{(k+1) \lambda}{t^{2}}, \frac{1}{2}\right)\right]}{k !}\left[\Gamma\left(\frac{1}{2}\right)-\left(\frac{(k+1) \lambda}{t^{2}}, \frac{1}{2}\right)\right] \\
& \alpha\left(1-\alpha^{e^{-\frac{\lambda}{x^{2}}}-1}\right)
\end{aligned}
$$

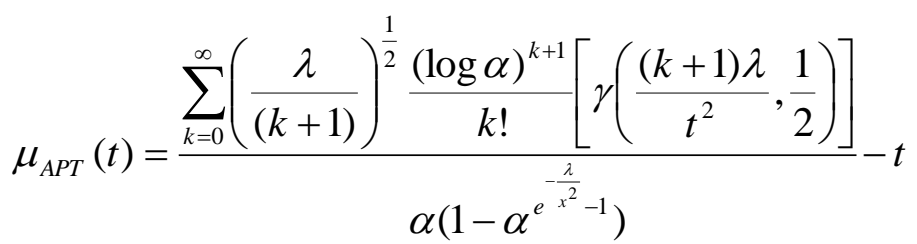

where

$$
\gamma(a, b)=\int_{0}^{\infty} u^{b-1} e^{-u} d u-\int_{a}^{\infty} u^{b-1} e^{-u} d u=\int_{0}^{a} u^{b-1} e^{-u} d u \quad \text { is }
$$

the incomplete gamma function.

Also the MWT is defined as

$$
\bar{\mu}_{A P T}(t)=t-\frac{1}{G_{A P T}(t)} \int_{0}^{t} x g_{A P T}(x) d x \text {. }
$$

On substituting eqn. (1) and (2) in eqn. (12) we get

$$
\bar{\mu}_{A P T}(t)=t-\frac{1}{\left(\alpha^{e^{-\frac{\lambda}{x^{2}}}}-1\right)}\left[\begin{array}{l}
\Gamma\left(\frac{(k+1) \lambda}{t^{2}}, \frac{1}{2}\right) \\
\sum_{k=0}^{\infty}\left(\frac{\lambda}{(k+1)}\right)^{\frac{1}{2}} \frac{(\log \alpha)^{k+1}}{(\alpha-1) k !}
\end{array}\right]
$$

\section{VII.ENTROPY}

The Renyi entropy for APIRD is given as

$$
R E_{X}(\rho)=\frac{1}{1-\rho} \log \left\{\begin{array}{l}
\sum_{k=0}^{\infty} \frac{(\log \alpha)^{k} \rho^{k}}{k !} 2^{\rho-1} \lambda^{\frac{1}{2}(1-\rho)} \\
\left(\frac{\log \alpha}{(\alpha-1)}\right)^{\rho} \frac{\Gamma\left(\frac{3}{2} \rho-\frac{1}{2}\right)}{(\rho+k)^{\left(\frac{3}{2} \rho-\frac{1}{2}\right)}}
\end{array}\right\}
$$

Where $\Gamma(a, b)=\int_{a}^{\infty} u^{b-1} e^{-u} d u$ is the incomplete gamma function.

\section{STRESS STRENGTH RELIABILITY}

Let $X_{1} \sim \operatorname{APIRD}\left(\alpha_{1}, \lambda_{1}\right)$ and $X_{2} \sim \operatorname{APIRD}\left(\alpha_{2}, \lambda_{2}\right)$ be two independent random variables then the stress strength eliability say $\mathrm{R}$ for APIRD has four cases discussed below: Case 1: $\alpha_{1} \neq 1$ and $\alpha_{2} \neq 1$

$$
\begin{aligned}
& R=\int_{0}^{\infty} g_{A P T 1}(x) G_{A P T 2}(x) d x . \\
& R=\frac{1}{\left(\alpha_{2}-1\right)}\left\{\sum_{k=0}^{\infty} \sum_{l=0}^{\infty} \frac{\left(\log \alpha_{2}\right)^{l}\left(\log \alpha_{1}\right)^{k+1}}{\left(\alpha_{1}-1\right) k ! l !(k+l+1)}-1\right\} .
\end{aligned}
$$

Case 2: $\alpha_{1}=1, \alpha_{2} \neq 1$.

$$
\begin{aligned}
& R=\int_{0}^{\infty} g_{1}(x) G_{A P T 2}(x) d x . \\
& R=\frac{1}{\left(\alpha_{2}-1\right)}\left\{\sum_{l=0}^{\infty} \frac{\left(\log \alpha_{2}\right)^{l}}{l !(l+1)}-1\right\} .
\end{aligned}
$$

Case 3: $\alpha_{1} \neq 1, \alpha_{2}=1$

$$
\begin{aligned}
R & =\int_{0}^{\infty} g_{A P T 1}(x) G_{2}(x) d x . \\
R & =\sum_{k=0}^{\infty} \frac{\left(\log \alpha_{1}\right)^{k+1}}{k !\left(\alpha_{1}-1\right)(k+2)} .
\end{aligned}
$$

Case 4: $\alpha_{1}=1, \alpha_{2}=1$

$$
\begin{aligned}
& R=\int_{0}^{\infty} \frac{2 \lambda_{1}}{x^{3}} e^{-\frac{\lambda_{1}}{x^{2}}} e^{-\frac{\lambda_{2}}{x^{2}}} d x . \\
& R=\left(\frac{\lambda_{1}}{\lambda_{1}+\lambda_{2}}\right) .
\end{aligned}
$$

\section{ORDER STATISTICS}

Let $x_{1}, x_{2}, \ldots, x_{n}$ be an ordered sample of size $\mathrm{n}$ from APIRD. Then the PDF of $X_{r}, X_{1}$ and $X_{n}$ is given as below

$$
\begin{aligned}
f_{r: n}(x)= & \frac{n !}{(n-r) !(r-1) !}\left[\frac{\alpha\left(1-\alpha^{e^{-\frac{\lambda}{x^{2}}}}\right)}{(\alpha-1)}\right]^{n-r}\left[\frac{\left(\alpha^{e^{-\frac{\lambda}{x^{2}}}}-1\right)}{(\alpha-1)}\right]^{r-1} \\
& \frac{\log \alpha}{(\alpha-1)} \frac{2 \lambda}{x^{3}} e^{-\frac{\lambda}{x^{2}}} \alpha^{e^{-\frac{\lambda}{x^{2}}}} .
\end{aligned}
$$


$f_{1: n}(x)=\frac{n}{(\alpha-1)^{n}} \log \alpha \frac{2 \lambda}{x^{3}} e^{-\frac{\lambda}{x^{2}}} \alpha^{n-1+e^{-\frac{\lambda}{x^{2}}}}\left(1-\alpha^{-\frac{\lambda}{x^{2}}}\right)^{n-1}$.

$f_{n: n}(x)=\frac{n}{(\alpha-1)^{n}} \log \alpha \frac{2 \lambda}{x^{3}} e^{-\frac{\lambda}{x^{2}}} \alpha^{-\frac{\lambda}{x^{2}}}\left(\alpha^{e^{-\frac{\lambda}{x^{2}}}}-1\right)^{n-1}$.

\section{QUANTILES AND SIMULATION}

The APIRD can easily be simulated by inverting eqn. (5) as follows: if $\mathrm{p}$ followsU $(0.1)$, then

$x=\left\{-\frac{1}{\lambda} \log \left[\frac{\log (1+p(\alpha-1))}{\log \alpha}\right]\right\}^{-\frac{1}{2}}$.

Quantiles are given as

$x_{q}=\left\{-\frac{1}{\lambda} \log \left[\frac{\log (1+q(\alpha-1))}{\log \alpha}\right]\right\}^{-\frac{1}{2}}$.

By putting $\mathrm{q}=0.5$ in above eqn. we obtain median

$x_{0.5}=\left\{-\frac{1}{\lambda} \log \left[\frac{\log (1+(0.5)(\alpha-1))}{\log \alpha}\right]\right\}^{-\frac{1}{2}}$.

\section{PARAMETER ESTIMATION}

Let $X_{1}, X_{2}, \ldots X_{n}$ be a random sample of size $\mathrm{n}$ from APIRD then the log likelihood function is given by $\log L=n \log 2+n \log \lambda+n \log (\log \alpha)-n \log (\alpha-1)$

$$
+n \log \left(\prod_{i=1}^{n}\left\{\frac{1}{x^{3}}\right\}\right)-\lambda \sum_{1=1}^{n} \frac{1}{x^{2}}+\sum_{1=1}^{n} e^{-\frac{\lambda}{x^{2}}} \log \alpha .
$$

Differentiating eqn. (14) w.r.t $\alpha$ and $\lambda$ respectively we get

$$
\begin{aligned}
& \frac{\partial}{\partial \alpha} \log L=\frac{n}{\alpha \log \alpha}-\frac{n}{(\alpha-1)}+\frac{\sum_{i=1}^{n} e^{-\frac{\lambda}{x_{i}^{2}}}}{\alpha} . \\
& \frac{\partial}{\partial \lambda} \log L=\frac{n}{\lambda}-\sum_{i=1}^{n} \frac{1}{x_{i}^{2}}-\sum_{i=1}^{n} e^{-\frac{\lambda}{x_{i}^{2}}} \frac{\log \alpha}{x_{i}^{2}} .
\end{aligned}
$$

On equating eqn. (15) and (16) to zero we obtain the MLE of $\alpha$ and $\lambda$. Techniques such as Newton-Raphson can be used to accomplish the task of estimating the parameters from eq. (15) and (16).

\section{XII.APPLICATION}

For accessing the flexibility of proposed model, the APIRD is compared with two well-established models by using two real life data sets. The models used for comparison are
- $\quad$ IRD with pdf given as

$$
g(x)=\left(\frac{2 \lambda}{x^{3}}\right) \exp \left(-\frac{\lambda}{x^{2}}\right) \quad x, \lambda>0 .
$$

- Weibull Distribution (WD) with pdf given as

$$
g(x)=\alpha \lambda(x \lambda)^{\alpha-1} \exp (-x \lambda)^{\alpha} \quad x, \alpha, \lambda>0 .
$$

For selecting the best fitted model, the values of log likelihood, AIC, AICC and BIC are computed for the proposed model and the models used for comparison and the model with minimum value of log likelihood, AIC, AICC and BIC is considered to be the best fitted for the given data sets .The software through which analysis is carried out is $\mathrm{R}$ software. The data sets used are:

Data set 1: This data set is discussed in Smith and Naylor [6].

Table 1 shows maximum likelihood estimates and different information measures for models. Figure 5(a) shows the plots of the estimated pdfs of APIRD and other competitive models.

Table 1: Estimates and performance of distribution

\begin{tabular}{|c|c|c|c|c|c|c|}
\hline \multirow{2}{*}{$\begin{array}{c}\text { Distribu } \\
\text {-tion }\end{array}$} & $\hat{\alpha}$ & $\hat{\lambda}$ & Log $\boldsymbol{l}$ & AIC & BIC & \multirow{2}{*}{ AICC } \\
\cline { 2 - 6 } APIRD & 120 & 751 & 392 & 788.15 & 792 & 788.32 \\
\hline IRD & & 2187 & 406 & 815.47 & 817 & 815.64 \\
\hline WD & 1.39 & 0.009 & 397 & 798.29 & 802 & 798.47 \\
\hline
\end{tabular}

Data set 2: This data set represents the relief times of twenty patients receiving an analgesic. This data set was taken from [7].

Table 1 shows maximum likelihood estimates and different information measures for models Figure 5(b) shows the plots of the estimated pdfs of APIRD and other competitive models.

Table 2: Estimates and performance of distribution

\begin{tabular}{|c|c|c|c|c|c|c|}
\hline \multirow{2}{*}{$\begin{array}{c}\text { Distribu } \\
\text {-tion }\end{array}$} & $\hat{\alpha}$ & $\hat{\lambda}$ & Log $\boldsymbol{l}$ & AIC & BIC & AICC \\
\cline { 2 - 6 } APIRD & 0.0042 & 6.26 & 15.6 & 35.2 & 37.24 & 35.9 \\
\hline IRD & & 2.76 & 21.1 & 44.3 & 45.36 & 45.0 \\
\hline WD & 2.787 & 0.46 & 20.5 & 45.1 & 47.16 & 45.8 \\
\hline
\end{tabular}



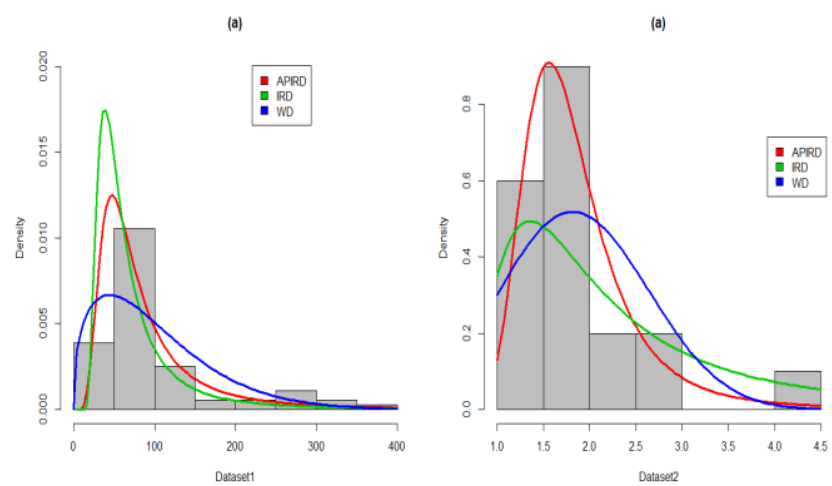

Figure 5. (a) Plots of the estimated PDF of APIRD and other competitive models for Data set 1. (b) Plots of the estimated PDF of APIRD and other competitive models for Data set 2.

\section{DISCUSSION}

Since APIRD has the minimum value of log likelihood, AIC, BIC and AICC. Hence APIRD can be considered to be best fit for the given data sets.

\section{CONCLUSION}

In this manuscript, we have proposed a new three parameter IRD based on APT known as APIRD. Explicit expression for moments, mgf., entropies, mean waiting time, mean residual time etc. has been derived. The parameter estimation has also been carried out. The real life applicability is exemplified by means of two real life data sets.

\section{REFERENCES}

[1] N. Trayer. Doklady Acad, Nauk, Belorus, U.S.S.R, 1964.

[2] R. Gh. Voda, (1972), "On the inverse Rayleigh variable", Rep. Stat. Apph. Res. Juse, Vol. 19, Issue., 4, pp.15-21, 1972.

[3] A. Mahadavi, D. Kundu, "A new method of generating distribution with an application to exponential distribution", Communications in Statistics-Theory and Methods, Vol. 46, Issue., 13, 2015.

[4] M. Nassar, A. Alzaatreh, M. Mead \&O. Abo-Kasem, "Alpha Power Weibull distribution: Properties and application", Communications in Statistics-Theory and Methods, Vol. 46, Issue., 20, 2017.

[5] A. S. Malik, S.P. Ahmad, "Alpha Power Rayleigh Distribution and Its Application to Life Time Data", International Journal of Enhanced Research in Management \& Computer Applications, Vol. 6, Issue., 11, pp. 212-219, 2017.

[6] R. L. Smith, J. C. Naylor, "A comparison of maximum likelihood and Bayesian estimators for the three-parameter Weibull distribution”, Appl Stat ,Vol. 36,pp. 358-369, 1987.

[7] A. J. Gross, V.A. Clark, "Survival distributions: Reliability applications in the biomedical sciences", Wiley-Interscience, New York, 1975.

[8] C. D. Devi, S. Thaddeus, "Information Retrieval Mechanism for Dynamic Health Care", International Journal of Computer Sciences and Engineering, Vol. 6 (9), pp. 105-107, 2018. 